\title{
CONDIÇÕES DE SAÚDE DE HOMENS IDOSOS COM OSTEOPOROSE
}

\author{
Jéssica da Silva Pinheiro, Ângela Oliveira, Bruna da Silva Pavan, Andréa Bona \\ Ughini, Matheus Santos Gomes Jorge e Lia Mara Wibelinger
}

UPF

*iessica.pinheiro88@hotmail.com

Acesso DOI: http://dx.doi.org/10.34059/ciejop.2019v27i1-9

\section{Resumo}

Introdução: A osteoporose é uma doença sistêmica caracterizada pela perda de massa óssea. Frequentemente associada a mulher, poucas evidências analisam o perfil de homens acometidos pela doença. Objetivo: analisar as condições de saúde de homens idosos com osteoporose. Metodologia: Estudo observacional descritivo, que avaliou 351 idosos, de ambos os sexos, residentes na zona urbana do município de Passo Fundo/RS. Os indivíduos responderam a um questionário estruturado, cujo mesmo abordou dados sobre o perfil sociodemográfico e o perfil de saúde dos entrevistados. O critérios de inclusão foram o sexo masculino, residentes da zona urbana do município de Passo Fundo/RS, capacidade de comunicar-se verbalmente com o entrevistador e que concordaram em assinar o Termo de Consentimento Livre e Esclarecido. Os critérios de exclusão foram indivíduos com acometimento auditivo ou disartria, residentes em instituições de longa permanência ou hospitalizados. Resultados: Dos 351 inicialmente recrutados, apenas 17 foram selecionados. A maioria tinha entre 60-69 anos, tinha baixo nível de escolaridade, era viúvo, residia com a família, não sofreu quedas ou hospitalizações no último ano, tinha dificuldade nas atividades diárias, realizava acompanhamento médico e fisioterapêutico, praticava atividade física e autoavaliou sua saúde como negativa. Conclusão: A descrição do perfil da amostra estudada pode auxiliar na compreensão da doença sobre este tema, encorajando a novas pesquisas com amostras mais robustas e ao fortalecimento das políticas públicas na saúde do homem, especialmente sobre a osteoporose nesta população.

Palavras-chave: Osteoporose; Perfil de Saúde; Saúde do homem

\section{Abstract}

Introduction: Osteoporosis is a systemic disease characterized by loss of bone mass. Often associated with women, there is little evidence of the profile of men affected by the disease. Objective: to analyze the health conditions of elderly men with osteoporosis. Methodology: Descriptive observational study, which evaluated 351 elderly, of both sexes, living in the urban area of Passo Fundo/RS. Individuals answered a structured questionnaire, which included data on the sociodemographic profile and the health profile of the interviewees. The inclusion criteria were male, residents of the urban area of the municipality of Passo Fundo/RS, ability to communicate verbally with the interviewer and who agreed to sign the Informed 
Consent Term. Exclusion criteria were individuals with auditory impairment or dysarthria, living in long-term institutions or hospitalized. Results: Of the 351 initially recruited, only 17 were selected. The majority were between 60-69 years old, had low level of education, were widowed, lived with family, did not suffered falls or hospitalizations in the last year, had difficulty in the activities of daily living, had poor visual health conditions, performed medical and physical therapy follow-up , practiced physical activity and self-assessed your health as negative. Conclusion: The description of the profile of the sample studied can help to understand the disease on this theme, encouraging new research with more robust samples and strengthening of public policies on men's health, especially on osteoporosis in this population.

Keywords: Osteoporosis; Health Profile; Men's Health

Introdução

O envelhecimento populacional é um fenômeno de interesse científico, pois há algum tempo ocorre uma transição demográfica caracterizada pelo aumento da expectativa de vida e redução das taxas de natalidade, fecundidade e mortalidade (Coutinho et al., 2014; Miranda et al., 2016). No Brasil, e em outros países subdesenvolvidos ou em desenvolvimento, são considerados idosos indivíduos com 60 anos ou mais, e em países desenvolvidos são considerados idosos indivíduos com 65 anos ou mais (World Health Organization, 2005).

As doenças crônicas não-transmissivveis são tidas como potenciais fatores para a redução da expectativa de vida e aumento de incapacidades em idosos (Campolina et al., 2013). Dentre estas, pode-se citar a osteoporose, uma doença sistêmica caracterizada pela redução da massa óssea e destruição da microarquitetura óssea. Como consequência, o indivíduo apresenta uma fragilidade mecânica e maior probabilidade de sofrer fraturas ao trauma mínimo. Embora todas as pessoas possam sofrer com a perda de massa óssea, as mulheres após a menopausa são as mais propensas a desenvolvê-la (Carvalho et al., 2004).

A etiologia da osteoporose é complexa e multifatorial. Dentre os fatores predisponentes e associados pode-se citar os demográficos (hereditariedade, etnia, idade avançada, sexo feminino), clínicos (baixo peso corporal, deficiência nutricional, deficiência hormonal), sociais (excessivo consumo de álcool, tabagismo, inatividade física), entre outros (Carvalho et al., 2004). 
Por muito tempo, a osteoporose foi considerada uma doença ligada exclusivamente ao sexo feminino, por conta da perda hormonal (estrógeno) nas mulheres pós-menopausa. Associado a isso, os níveis funcionais do hormônio masculino (testosterona) persistem até a sétima década de vida. Com o avançar do tempo, novos fatores de risco na vida moderna surgiram com o envelhecimento populacional evidenciando a osteoporose masculina (Oliveira e Guimarães, 2010).

A saúde óssea dos idosos pode ser beneficiada por simples mudanças nos hábitos de vida, como uma alimentação balanceada e a prática regular de exercício físico. Contudo, estas ações nem sempre são conhecidas pela população em massa (Carvalho et al., 2004), especialmente pelos homens que, culturalmente, são o público que menos presta atenção na sua saúde em relação às mulheres.

Poucos estudos abordam condições sociodemográficas e clínicas de homens com osteoporose, em especial pela falta de políticas públicas que discutem o tema abordado. Neste sentido, o objetivo do presente estudo foi analisar as condições de saúde de homens idosos com osteoporose.

\section{Metodologia}

Estudo quantitativo, descritivo de corte transversal, cujo mesmo faz parte de um projeto denominado "Caracterização das condições de saúde dos idosos do município de Passo Fundo/RS", aprovado pelo Comitê de Ética em Pesquisa em Seres Humanos da Universidade de Passo Fundo, sob o parecer $n^{\circ}$ 447/2010 e que está em consonância com a resolução 196/96 do Conselho Nacional de Saúde.

Avaliaram-se 351 idosos por meio de uma amostragem aleatória simples. Como critérios de inclusão para o estudo foram selecionados idosos do sexo masculino, residentes da zona urbana do município de Passo Fundo/RS, com capacidade de comunicar-se verbalmente com o entrevistador e que concordaram em assinar o Termo de Consentimento Livre e Esclarecido, mediante prévia explicação e esclarecimento de dúvidas. Os critérios de exclusão foram para indivíduos com acometimento auditivo ou disartria, residentes em instituições de longa permanência ou hospitalizados. 
Os participantes responderam a um questionário estruturado que foi elaborado e adaptado pelos próprios pesquisadores. Analisaram-se os itens referentes à caracterização sociodemográfica (gênero, faixa etária, escolaridade, estado civil e com quem residia em seu lar) e as condições de saúde (ocorrência de quedas, dificuldade nas atividades de vida diária, consultas regulares ao médico, internação hospitalar, se realiza/realizou fisioterapia, se é praticante de atividade física e a autopercepção de saúde).

A coleta de dados foi realizada, entre agosto de 2011 e setembro de 2012, por um grupo de acadêmicos do curso de Fisioterapia da Universidade de Passo Fundo, em locais públicos da zona urbana do município de Passo Fundo/RS. Ao fim, as informações foram tabuladas em um software estatístico e realizada a análise estatística descritiva.

\section{Resultados}

Dos 351 indivíduos inicialmente entrevistados, apenas 17 foram identificados como homens com osteoporose $(4,84 \%$ da amostra inicial). Todos estes indivíduos preencheram os critérios de inclusão do presente estudo e tiveram seus dados analisados. A Tabela 1 apresenta a caracterização sociodemográfica da amostra estudada.

Tabela 1. Caracterização sociodemográfica de homens idosos com osteoporose. Passo Fundo/RS, 2019

\begin{tabular}{lc}
\hline Variáveis & $\mathrm{N}(\%)$ \\
\hline Faixa etária & $09(52,9)$ \\
$60-69$ anos & $05(29,4)$ \\
$70-79$ anos & $03(17,6)$ \\
$80-89$ anos & $15(88,2)$ \\
Escolaridade & $02(11,8)$ \\
Ensino fundamental incompleto ou completo & \\
Ensino médio incompleto ou completo & $07(41,2)$ \\
Estado civil & $09(52,9)$ \\
Casado & $01(5,9)$ \\
Viúvo & \\
Divorciado / Separado & $09(52,9)$ \\
Moradia & $08(47,1)$ \\
Mora com familiares & \\
Mora sozinho & \\
\hline
\end{tabular}


Legenda: N (valor absoluto); \% (valores relativo)

Ao analisar as características sociodemográficas, verificamos que a maioria dos idosos concentra-se na faixa etária entre 60-69 anos, apresenta baixo nível de escolaridade (ensino fundamental incompleto ou completo), era composta por viúvos e residia com familiares. A Tabela 2 apresenta as condições de saúde dos indivíduos da amostra estudada.

Tabela 2. Condições de saúde de homens idosos com osteoporose. Passo Fundo/RS, 2019

\begin{tabular}{lc}
\hline Variáveis & $\mathrm{N}(\%)$ \\
\hline Quedas & $05(29,4)$ \\
Sim & $12(70,6)$ \\
Não & $12(70,6)$ \\
Dificuldades para realizar atividades de vida diárias & $05(29,4)$ \\
Sim & \\
Não & $14(82,4)$ \\
Consulta regular ao médico & $03(17,6)$ \\
Sim & \\
Não ou apenas quando adoece & $07(41,2)$ \\
Internação hospitalar no último ano & $10(58,8)$ \\
Sim & \\
Não & $11(64,7)$ \\
Fisioterapia & $06(35,3)$ \\
Sim & \\
Não & $14(82,4)$ \\
Atividade física & $03(17,6)$ \\
Sim & \\
Não & $11(64,7)$ \\
Autopercepção de saúde & $06(35,3)$ \\
Negativa (péssima, ruim ou regular) & \\
Positiva (ótimo ou boa) &
\end{tabular}

Legenda: N (valor absoluto); \% (valor relativo)

As condições de saúde dos idosos entrevistados mostram que a maioria não sofreu qualquer tipo de queda, porém tem dificuldades para realizar atividades diárias. Ainda, os idosos realizavam consultas regulares ao médico, não tiveram internação hospitalar no último ano, praticavam atividade física, realizavam acompanhamento fisioterapêutico e autoavaliaram sua saúde como negativa. 


\section{Discussão}

Por muito tempo, a osteoporose foi considerada uma doença exclusivamente do sexo feminino. Porém, esta ideia sofreu modificações e atualmente é reconhecida como um problema de saúde pública, em virtude do aumento significativo da incidência de fraturas osteoporóticas nos homens (Oliveira, 2002). O que justifica a necessidade da realização do presente estudo, onde observamos que os homens idosos com osteoporose apresentaram pontos sociodemográficos (baixo nível de escolaridade e condição de viuvez) e clínicos (dificuldades para realizar atividades diárias e autopercepção de saúde negativa) considerados potenciais fatores que influenciam para uma saúde prejudicada de indivíduos na terceira década de vida.

Um estudo investigou fatores associados à osteoporose em mulheres, onde constataram que a baixa escolaridade foi um fator associado à osteoporose na população (Costa et al., 2016). Embora nosso estudo tenha investigado as condições de saúde de homens idosos com osteoporose constatamos que nossa amostra também apresentou predomínio de baixa escolaridade. Ademais, os autores supracitados (Costa et al., 2016) relataram, ainda, que na literatura até aquele momento não foram identificados estudos com estes mesmos achados e que esta associação entre baixa escolaridade e osteoporose possa ter sido em virtude do efeito que a educação exerce no estilo de vida, na nutrição e na condição econômica (Keramat et al., 2008).

Em nossos resultados demonstramos que a maioria dos idosos com osteoporose era viúva, o que concorda com o estudo de Costa et al. (2016), onde os autores verificaram que ter uma união estável foi um fator protetor para a osteoporose. Uma de nossas hipóteses para isso, é que nossa amostra é composta exclusivamente pelo público masculino que, culturalmente, presta menor atenção aos cuidados relacionados à própria saúde. Em uma situação de viuvez, provavelmente a falta de um cônjuge possa contribuir para que este problema seja agravado, pois, historicamente, a mulher era o membro que cuidava da família (marido e filhos), em diversas instâncias como educação, moradia e, inclusive, da saúde. 
Nossos resultados apontam para um alto índice de idosos com dificuldade para realizar as atividades de vida diária, os quais podem ser explicados pelo fato de que, as consequências oriundas da osteoporose, principalmente as fraturas por ela causadas, contribuem para diversos problemas na saúde dos indivíduos acometidos pela doença, incluindo a dificuldade para realizar as atividades de vida diária (Sato et al., 2014). Nossos resultados sobre o impacto das atividades de vida diária em idosos com osteoporose são reforçados pelos achados de outro estudo onde os pesquisadores identificaram que a dificuldade para realizar as atividades de vida diária em idosos sem osteoporose foi baixa (15,7\%) (Andrade, 2018).

Algumas referências investigaram os fatores associados à osteoporose em idosos brasileiros e identificou-se que a autopercepção de saúde negativa (regular, ruim ou péssima) foi um dos fatores associados à doença (Baccaro et al., 2013; Rodrigues e Barros, 2016). Isso corrobora com nosso resultado onde observamos que a maioria dos homens idosos com osteoporose autoperceberam sua saúde como negativa (regular, ruim ou péssima).

Outros pontos sociodemográficos (idade mais jovem e residir com familiares) e clínicos (não sofrer quedas, realizar acompanhamento médico e fisioterapêutico, não terem internado em hospital e praticar atividade física) que foram relatados pelos idosos são tidos como potenciais fatores que podem influenciar positivamente para uma boa saúde na terceira década de vida.

Nossos dados referentes a maioria da amostra ser composta por homens idosos com 60-69 anos assemelha-se a outro estudo brasileiro que verificou a prevalência e fatores associados à osteoporose em mulheres, onde os autores observaram que a idade média dos participantes foi de 64,1 anos (Baccaro et al., 2013). Já outro estudo que teve o mesmo objetivo e avaliou idosos de ambos os sexos verificou que idosos com 80 anos ou mais apresentaram maior prevalência de osteoporose (Rodrigues e Barros, 2016), indo de encontro aos nossos achados. Contudo essa inferência deve ser realizada com cautela, uma vez que no primeiro estudo (Baccaro et al., 2013) foram avaliadas só mulheres e no segundo estudo (Rodrigues e Barros, 2016) idosos de ambos os sexos, sendo que quase metade da amostra era composta por homens. Ainda, outra informação importante deve-se ao fato de que mulheres apresentam perda óssea muito mais cedo que os homens em 
virtude da sua produção hormonal finalizar após a menopausa e dos homens aproximadamente após os 70 anos (Oliveira e Guimarães, 2010).

Em nosso estudo observamos que a maioria dos homens idosos com osteoporose moravam com seus familiares. Em um estudo que avaliou os fatores associados à condição de moradia (morar sozinho ou não) de idosos, a osteoporose não foi um fator associado, mas o sexo feminino sim (Cauduro e Gonçalves; 2013). O que pode justificar nosso achado, pois a amostra do estudo atual foi composta exclusivamente por homens idosos.

Um estudo realizado com idosos brasileiros residentes no município de Campinas/SP, demonstrou que entre os fatores associados à osteoporose nesta população está a ocorrência de quedas nos últimos 12 meses (Rodrigues e Barros, 2016). Esse resultado vai de encontro ao nosso estudo, pois os idosos com osteoporose não apresentaram uma elevada prevalência de quedas. Além disso, é importante reconhecer que a prevalência de quedas é no sexo feminino, como observado em um estudo com idosos do Sul do Brasil (Vieira et al., 2018), aspecto este que pode ter influenciado em nosso achado, pois nossa variável era composta apenas por homens idosos.

Idosos com osteoporose devem ser acompanhados regularmente. Recentemente foram delineadas as diretrizes brasileiras para o diagnóstico e tratamento da osteoporose em mulheres na pós-menopausa, no qual são explanadas as estratégias medicamentosas e não medicamentosa para este público (Radominski et al., 2017). Isso demonstra a necessidade de acompanhamento médico para indivíduos com osteoporose, assim como os idosos estudados a fizeram. Todavia, isso reafirma a fragilidade nas políticas públicas para atenção a saúde de homens com osteoporose, pois até o presente momento não foram delineadas diretrizes brasileiras para este público.

Um estudo analisou os dados do DATASUS do triênio 2008-2010 buscando traçar um perfil sobre o tratamento de indivíduos com osteoporose e fraturas delas decorrentes no Sistema Único de Saúde (SUS). Observou-se que foram realizados 3.252.756 procedimentos (96,4\% em ambulatórios e $70,4 \%$ em hospitais), que totalizaram $\mathrm{R} \$ 288.986 .335,15$ de despesas aos cofres públicos. A maioria dos usuários eram mulheres (naturalmente em virtude da maior prevalência de Fórum Internacional de Qualidade de Vida e Saúde - Curitiba, 15 de junho de 2019 
osteoporose neste sexo), na faixa etária de 60-69 anos (46,3\%) (Moraes et al., 2014). Neste prisma, observamos que houve um equilíbrio entre a prevalência de indivíduos que foram internados $(41,2 \%)$ e não foram internados $(58,8 \%)$ no último ano, ressaltando que nossos indivíduos eram apenas homens, mas estavam concentrados, em sua maioria, na faixa etária dos 60-69 anos.

A fisioterapia é essencial para idosos com osteoporose, especialmente pela sua abordagem de caráter preventivo, evitando quedas e fraturas, avanço da perda óssea e incentivo a alimentação balanceada e a atividade física. Além disso, outros objetivos incluem a reestruturação óssea (em caso das fraturas), melhora da força e qualidade muscular, da amplitude de movimento articular, do equilíbrio, da coordenação da postura, da marcha, da funcionalidade, da qualidade de vida, entre outras (Wibelinger, 2014). O que corrobora com nossos achados, visto que a maioria dos idosos com osteoporose realizavam fisioterapia para tratamento da sua doença.

A inatividade física é um fator de risco para o desenvolvimento de osteoporose (Costa et al., 2016). Todavia, nossos resultados apontaram que os homens idosos com osteoporose eram, na sua maioria, praticantes de atividade física. Isso pode ser explicado pelo fato de que muitos idosos procuram a atividade física com caráter curativo aos problemas de saúde já instalados e não como forma de prevenção (Gomes e Zazá, 2009).

Nosso estudo pode apresentar limitações. O pequeno número amostral não nos permitiu realizar análises estatísticas que verificassem a associação das variáveis independentes com a nossa variável dependente (osteoporose). Porém isso não diminui o valor científico de nossa análise para compreender as condições de saúde dessa população e permitir a geração de dados que possam auxiliar na discussão de pesquisas futuras.

\section{Conclusão}

Os homens idosos com osteoporose deste estudo concentram-se na faixa etária entre 60-69 anos, têm escolaridade básica, são viúvos e residem com familiares. Ainda, os idosos têm dificuldades para realizar atividades diárias, 
realizam consulta regular ao médico, praticam atividade física, realizam fisioterapia e tem autopercepção de saúde negativa.

Ainda, a descrição do perfil da amostra estudada pode auxiliar na compreensão da doença sobre este tema, incentivando a novas pesquisas com amostras mais robustas e ao reforço de políticas públicas da saúde do homem, especialmente sobre a osteoporose nesta população.

\section{Referências}

ANDRADE, Fabíola Bof. Inequalities in basic activities of daily living among older adults: ELSI-Brazil, 2015. Revista de Saúde Pública, São Paulo, v. 52, supl. 2, n. 14, 2018.

BACCARO, Luiz Francisco; MACHADO, Vanessa de Sousa Santos; COSTA-PAIVA, Lúcia; SOUSA, Maria Helena; OSIS, Maria José; PINTO-NETO, Aarão Mendes. Factors associated with osteoporosis in Brazilian women: a population-based household survey. Archives of osteoporosis. v. 8, n. 138, p. 1-1, 2013.

CAMPOLINA, Alessandro Gonçalves; ADAMI, Fernando; SANTOS, Jair Licio Ferreira; LEBRÃO, Maria Lúcia. A transição de saúde e as mudanças na expectativa de vida saudável da população idosa: possíveis impactos da prevenção de doenças crônicas. Cadernos de Saúde Pública, v. 29, n. 6, p.1217-1229, 2013.

CARVALHO, Cecília Maria Resende Gonçalves de; FONSECA, Carla Cristina Carvalho; PEDROSA, José Ivo. Educação para a saúde em osteoporose com idosos de um programa universitário: repercussões. Cadernos de Saúde Pública, Rio de Janeiro, v. 20, n. 3, p. 719-726, 2004.

CAUDURO, Adroaldo; GONÇALVES, Ângelo José; CAUDURO, Maria Heloísa Fialho. Fatores associados a morar sozinho e suas diferenças regionais em idosos residentes de Porto Alegre e Manaus. Estudos Interdisciplinares do Envelhecimento. Porto Alegre, v. 18, n. 2, p. 349-365, 2013.

COSTA, Angra Larissa Durans. Osteoporose na atenção primária: uma oportunidade para abordar os fatores de risco. Revista Brasileira de Reumatologia. São Paulo, v. 56, n. 2, p. 111-116, 2016.

COUTINHO, Renato Xavier; RODRIGUES, Juliana Maria; ACOSTA, Marco Aurélio de Figueiredo. Educação e envelhecimento: realidades da educação de jovens e adultos. Revista Brasileira de Ciências do Envelhecimento Humano, Passo Fundo, v. 11, n. 1, p.33-45, 2014.

GOMES, Kátia Virgínia; ZAZÁ, Daniela Coelho. Motivo de adesão à prática de atividade física em idosas. Revista Brasileira de Atividade Física e Saúde, v. 14, n. 2, p. 969984, 2009. 
KERAMAT, Afsaneh; PATWARDHAN, Bhushan; LARIJANI, Bagher; CHOPRA, Arvind; MITHAL, Ambrish; CHAKRAVARTY, Devlina; ADIBI, Hossein; KHOSRAVI, Ahmad. The assessment of osteoporosis risk factors in Iranian women compared with Indian women. BMC Musculoskeletal Disorders, v. 9, n. 28, p. 1-1, 2008.

MIRANDA,Gabriella Morais Duarte; MENDES Antonio da Cruz Gouveia; SILVA, Ana Lucia Andrade da. $O$ envelhecimento populacional brasileiro: desafios e consequências sociais atuais e futuras. Revista Brasileira de Geriatria e Gerontologia. Rio de Janeiro, v.19, n.3, p.507-519, 2016.

MORAES, Luci Fabiane Scheffer; SILVA, Everton Nunes; SILVA, Diego Augusto Santos; PAULA, Ana Patrícia de. Gastos com o tratamento da osteoporose em idosos do Brasil (2008 - 2010): análise dos fatores associados. Revista Brasileira de Epidemiologia. São Paulo, v. 17, n. 3, p. 719-734, 2014.

OLIVEIRA, Lindomar Guimarães. Osteoporose - Guia para Diagnóstico, Prevenção e Tratamento. Rio de Janeiro: Revinter; 2002.

OLIVEIRA, Lindomar Guimarães; GUIMARÃES, Mara Lucia Rassi. Osteoporose no homem. Revista Brasileira de Ortopedia., São Paulo , v. 45, n. 5, p. 392-396, 2010.

SATO, Masayo; VIETRI, Jeffrey; FLYNN, Jennifer A.; FUJIWARA, Saeko. Bone fractures and feeling at risk for osteoporosis among women in Japan: patient characteristics and outcomes in the National Health and Wellness Survey. Archives of osteoporosis. v. 9, n. 199, p. 1-1, 2014.

VIEIRA, Luna S.; GOMES, Ana Paula; BIERHALSL, Isabel O.; FARÍAS-ANTÚNEZ, Simone; RIBEIRO, Camila G.; MIRANDA, Vanessa A.; LUTZL, Bárbara H.; BARBOSA, Thiago G. S.; LIMA, Natália P.; BERTOLDI, Andréa D.; TOMASIL, Elaine. Quedas em idosos no Sul do Brasil: prevalência e determinantes. Revista de Saúde Pública. v. 52, n. 22, 2018.

WIBELINGER, Lia Mara. Fisioterapia em Reumatologia. $2^{\text {a }}$ ed. Rio de Janeiro: Revinter; 2014.

WORLD HEALTH ORGANIZATION. Envelhecimento ativo: uma política de saúde. Brasília: Organização Pan-Americana da Saúde Brasil. 2005. Disponível em: $<$ http://bvsms.saude.gov.br/bvs/publicacoes/envelhecimento ativo.pdf $>$. Acesso em 10 abr 2019. 